\title{
Optimization of Lobed Perforated Panel Diffuser: Numerical Study of Orifice Geometry
}

\author{
Amina Meslem ${ }^{1}$, Florin Bode ${ }^{2,3}$, Ilinca Nastase ${ }^{2} \&$ Olivier Martin $^{1}$ \\ ${ }^{1}$ LaSIE, University of La Rochelle, Pôle Sciences et Technologie, Avenue Michel Crépeau, La Rochelle, France \\ ${ }^{2}$ CAMBI, Technical University of Civil Engineering in Bucharest, Building Services Department, 66 Avenue \\ Pache Protopopescu, Bucharest, Romania \\ ${ }^{3}$ Technical University of Cluj-Napoca, Mechanical Engineering Department, B-dul Muncii nr.103-105, D03, \\ Cluj-Napoca, Romania
}

Correspondence: Meslem Amina, LaSIE, University of La Rochelle, Pôle Sciences et Technologie, Avenue Michel Crépeau, La Rochelle 17042, France. Tel: 33-5-4645-8385. E-mail:ameslem@univ-lr.fr

Received: October 23, 2012

doi:10.5539/mas.v6n12p59
Accepted: November 13, 2012 Online Published: November 21, 2012

URL: http://dx.doi.org/10.5539/mas.v6n12p59

\begin{abstract}
Passive control of jet flows in order to enhance mixing and entrainment is of wide applicative interest. Our purpose is to develop new air diffusers for Heating Ventilating Air Conditioning systems, by using lobed diffusers, in order to ameliorate the users' thermal comfort. For a lobed perforated panel as a diffuser (Meslem et al., 2010), the jet induction optimization will be made by means of optimizing elementary orifice geometry and by arrangement of the neighboring orifices on the perforated panel. An experimental and a numerical investigation using different turbulence models has been carried out over the flow field of turbulent twin cross-shaped jet, in a recent study (Meslem et al., 2011). In comparison to Particle Image Velocity measurements, it was shown that among the investigated viscous models, the SST k- $\omega$ turbulence model is able to reproduce reasonably well ambient air induction, jets interaction and global expansion of the studied jets when the flow is resolved by numerical simulation through the lobed diffuser. In this study the SST k- $\omega$ turbulence model is used for the numerical simulations of parallel cross-shaped jets, based on the previous experimental validation. This work is dedicated to the lobe shape effect on flow dynamics and induction performance of the twin cross-shaped jet. It was shown that the lobes with rounded peaks are more advantageous than the right angled lobes.
\end{abstract}

Keywords: lobed jet, geometry optimization, CFD, parallel jets

\section{Introduction}

An innovation for optimizing air diffusion with application in HVAC systems (Heating, Ventilation, and Air Conditioning) by means of passive control of air jets through lobed diffusers was presented in previous studies (Meslem et al., 2010; Nastase et al., 2011). In this studies it was shown that a cross-shaped jet issued from a perforated panel with lobed orifices, has the capability of inducing twice as much ambient air as a circular jet from a perforated panel when the spacing between orifices is $S=3.5 D_{e}\left(D_{e}\right.$ is the equivalent diameter based on the exit area of the elementary orifice). The fact is that the for both studied flows, cross and circular, values for streamwise maximum velocities are comparable in the far field, which suggest comparable throws for the two studied cases, despite the consistent gain in ambient air induction for the cross-shaped perforated panel flow.

The induction performance of each type of perforated panels is depends largely by geometrical parameters that influence the dynamics of each elementary jet and by the interaction of the large scale flow structures generated by each individual orifice. The development of these streamwise large scale structures must not be affected by the interaction of neighboring parallel lobed jets (Figure 1a), because these structures have a dominant effect on induction phenomenon (Nastase et al., 2008; Meslem et al., 2011). Of different geometrical parameter which may influence the jet flow dynamics and ambient air induction is the lobe shape of the lobed orifice. In this work, we compare twin cross-shaped orifices with straight lobes (Figure 1b) to twin cross-shaped orifices with rounded lobes (Figure 1c) on jet flow dynamics and entrainment for a fixed orifice spacing $S=2 D_{e}$. The reference case analysed in the previous paper (Meslem et al., 2011) is considered and compared to a modified case. In the new case, all geometrical parameters of the diffuser are conserved, except the shape of the top of the lobes of the 
orifice. A rounded lobe (Figure 1c) is compared to a reference straight lobe (Figure 1b).

In a previous study (Meslem et al., 2011) the performance of three turbulence models (SST k- $\omega$, Reynolds Stress Model (RSM) and standard k- $\varepsilon$ model) was investigated. The general conclusion was that none of these viscous models could predict reasonably well all flow characteristics.

However, it was shown that among the investigated viscous models, the SST k- $\omega$ turbulence model is able to reproduce reasonably well ambient air induction, jets interaction and global expansion of the studied jets when the flow is resolved by numerical simulation through the lobed diffuser.

In this study, relying on the previous results, the SST k- $\omega$ turbulence model is taken into account for designing the optimal lobe shape of the twin orifice diffuser. Knowing the imperfect prediction of the SST k- $\omega$ turbulence model, even in the case of the best predicted dynamic quantities (e.g. the gap between the prediction and measurements of the streamwise evolution of jet volumetric flow rate was up to 14\%) (Meslem et al., 2011), we are looking here to find some trends rather than detailed evaluations of these quantities. These trends would be functions of the considered geometrical parameter.

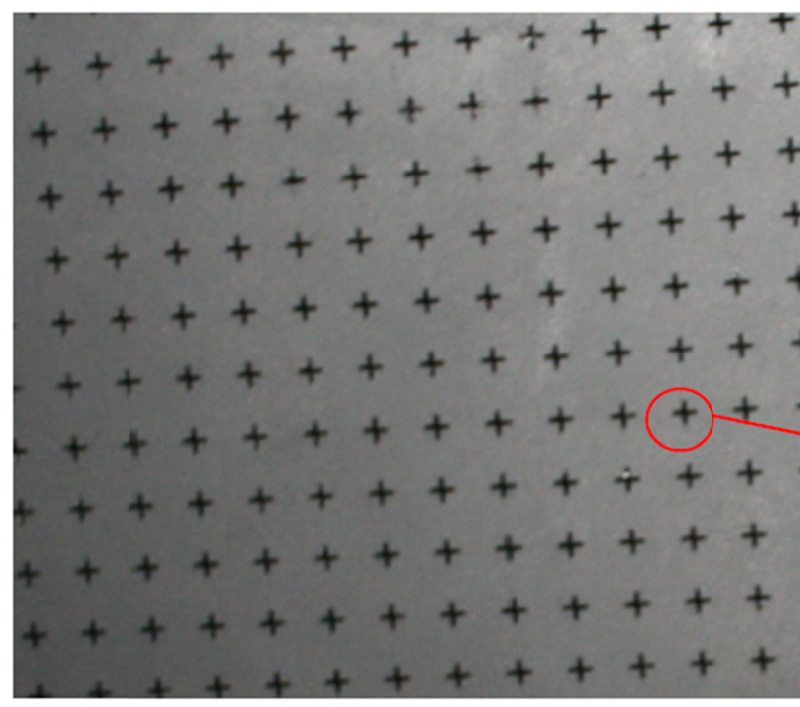

(a)

(b)

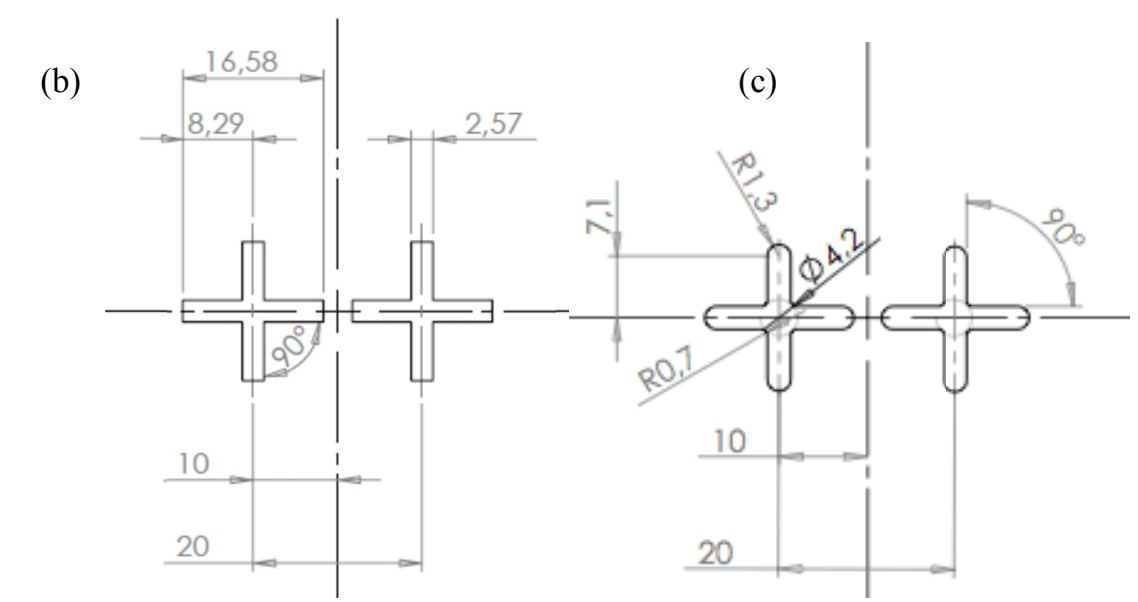

Streamwise structures and the resulting axis-switching phenomenon

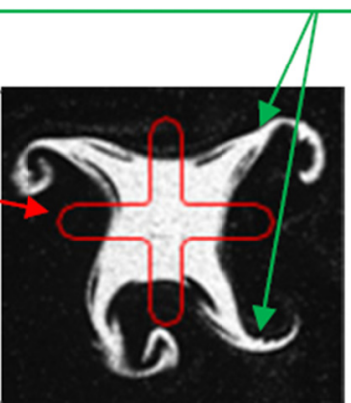

Figure 1. (a) The lobed perforated panel diffuser; (b) Twin cross orifices with right angled lobes (Case 1: LL O1 $-S=2 D_{e}$ ); (c) Twin cross orifices with rounded lobes (Case 2: LL O2 $-S=2 D_{e}$ )

The results are intended to optimize the lobed perforated panel diffuser for HVAC applications. In the future, the performance of the designed diffuser will be analyzed experimentally in the full-scale model room with controlled thermal environment, recently constructed in the French Laboratory of La Rochelle (LaSIE). We plan to study the influence of this innovative perforated panels used as a mixing ventilation diffuser in the full-scale model room over the thermal and draft comfort in the occupied zone and around a thermal mannequin body (Croitoru, 2011; Croitoru et al., 2011). 


\section{Materials and Methods}

\subsection{Orifice Jet Geometry and Jet Flow Visualization}

The air jet studied numerically is generated using twin cross-shaped orifices (Figure $1 \mathrm{~b}$ and c). The equivalent diameter (based on the exit area) of each elementary orifice is $D_{e}=10 \mathrm{~mm}$ and the distance between the orifices centres is $S=20 \mathrm{~mm}=2 D_{e}$. Two configurations are considered and compared: (1) cross-shaped orifices with straight lobes (Figure 1b), previously simulated and validated with experimental data (Meslem et al., 2011) and (2) cross-shaped orifices with rounded lobes (Figure 1c). In the two cases, the initial Reynolds number based on the mean exit velocity and on the equivalent diameter $D_{e}$ is around 3000 (Table 1).

Table 1. Initial flow conditions

\begin{tabular}{ccccccccc}
\hline $\begin{array}{c}\text { Studied } \\
\text { Case }\end{array}$ & $\begin{array}{c}\text { Turbulence } \\
\text { model }\end{array}$ & $\begin{array}{c}\text { Number of } \\
\text { cells }\end{array}$ & $\begin{array}{c}Q_{0} \\
\left(\mathrm{~m}^{3} / \mathrm{s}\right)\end{array}$ & $\begin{array}{c}U_{\text {Omean }} \\
(\mathrm{m} / \mathrm{s})\end{array}$ & $\begin{array}{c}U_{\text {OJC }} \\
(\mathrm{m} / \mathrm{s})\end{array}$ & $\begin{array}{c}U_{0 m} \\
(\mathrm{~m} / \mathrm{s})\end{array}$ & $\begin{array}{c}U_{\text {omean }} D_{e} / v \\
-\end{array}$ & $\begin{array}{c}U_{0 J C} D_{e} / v \\
-\end{array}$ \\
\hline $\begin{array}{c}\text { Case 1: } \\
\text { LL O1 }\end{array}$ & $\begin{array}{c}S S T k-\omega \\
\text { model }\end{array}$ & $\begin{array}{l}4 \text { million } \\
S=2 D_{e}\end{array}$ & $7.21 \times 10^{-4}$ & 4.58 & 6.03 & 6.27 & 2924 & 3841 \\
Case 2: & & & & & & & & \\
LL O2 & $\begin{array}{c}S S T k-\omega \\
\text { model }\end{array}$ & 5 million & $7.21 \times 10^{-4}$ & 4.58 & 5.47 & 6.07 & 2924 & 3484 \\
$S=2 D_{e}$ & & & & & & & \\
\hline
\end{tabular}

We are questioning ourselves on the influence of the shape of the lobe peak on the near exit jet development. Is this minimal change of the lobe shape having some influence on the dynamical quantities of the flow? We have to note here that the distance between the centres of two adjacent orifices $S=2 D_{e}$ is conserved. The change in the shape of the lobe peak is slightly modifying the distance s between the two neighboring peaks. Indeed, this distance is $s=3.2 \mathrm{~mm}$ for the orifices with rounded lobes, and respectively, $s=3.4 \mathrm{~mm}$ for the right angled lobes. This way, the variation related to the s parameter, for the two cases, is relatively reduced, being $0.2 \mathrm{~mm}=2 \% D_{e}$. Therefore, if there is any difference that has to be observed between the two studied flows, we could consider that the shape of the lobes is mainly the responsible geometrical parameter. We have also to note here that the volumetric flow rate at the inlet is the same in the two configurations, resulting in the same mean exit velocity $\mathrm{U}_{0 \text { mean }}$ and the same exit Reynolds number based on this velocity (Table 1).

The questioning related to the influence of the shape of the lobe came to us from the observation of Figure 2. The images of the transverse plane of the two flows at $X=0.5 D_{e}$ (Figure $2 \mathrm{a}, \mathrm{b}$ and c) displays an interesting phenomenon. Indeed in the case of the right angled lobes, a "pinch" of the flow is observable both in elementary (Figure 2b) and twin jet (Figure 2a) configuration. It follows the development of a streamwise structure at the peak of each lobe, a phenomenon which is not visible if the peak of the lobe has a rounded shape (Figure 2c). 
(a)

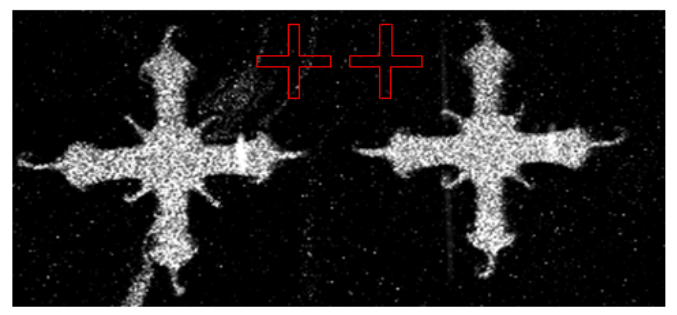

(d)

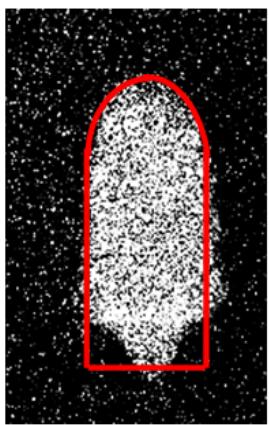

(b)

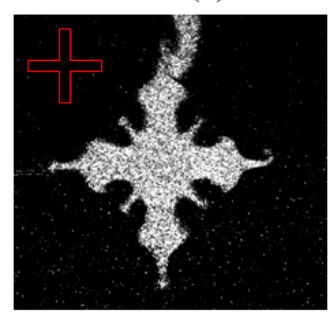

(c)

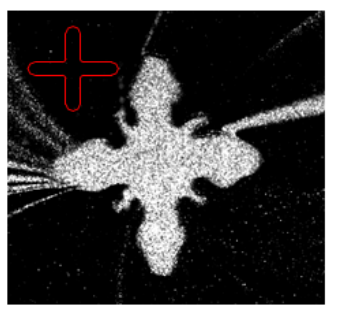

Figure 2. Flow visualizations at $X=0.5 D_{e}$, from different orifices: (a) twin cross-shaped orifices with straight lobes(LLO1); (b) elementary cross-shaped orifice with straight lobes (O1); (c) elementary cross-shaped orifice with rounded lobes $(\mathrm{O} 2)$; (d) elementary "door-shaped" orifice

This observation brought us to build a quasi rectangular orifice having one rounded extremity and one right angled extremity (Figure 2d). On the image corresponding to the flow generated by this orifice, that we will call "door shaped orifice", the phenomenon described previously is clearly observable: the flow is "pinched" on the right angled extremity and it is conserving the shape imposed by the orifice on the rounded extremity.

\subsection{Numerical Simulation of the Twin Jet}

\subsubsection{Governing Equations}

The Reynolds-Averaged Navier-Stokes (RANS) equations for the mass and momentum for an incompressible and isothermal flow can be written in tensor notation as follows:

$$
\begin{gathered}
\frac{\partial}{\partial X_{i}}\left(<U_{i}>\right)=0 \\
\frac{\partial\left(<U_{i}><U_{j}>\right.}{\partial X_{j}}=-\frac{1}{\rho} \frac{\partial<p>}{\partial X_{i}}+\frac{\partial}{\partial x_{j}}\left(v\left(\frac{\partial<U_{i}>}{\partial X_{j}}+\frac{\partial<U_{j}>}{\partial X_{i}}\right)\right)+\frac{\partial}{\partial X_{j}}\left(-<u_{i}^{\prime} u_{j}^{\prime}>\right)
\end{gathered}
$$

where $<U_{i}>$ is the mean velocity of the flow, $<p>$ is the mean pressure, $\rho$ is the density of the studied fluid and $v$ is the kinematic viscosity of the fluid. The mean values of the velocity fluctuating tensors $\left\langle u_{i}^{\prime} u_{j}^{\prime}\right\rangle$ in Eq. (2) are unknown. This term is identified as Reynolds stresses.

In this study, we used the SST k- $\omega$ RANS closure to model these terms. This model is chosen because in our previous study (Meslem et al., 2011) it was shown that among three different viscous models (k-€ standard, SST $\mathrm{k}-\omega$, and RSM), SST k- $\omega$ turbulence model is able to reproduce reasonably well ambient air induction, jets interaction and global expansion of the studied jets when the flow is resolved by numerical simulation through the lobed diffuser. The SST k- $\omega$ turbulence model is well known and widely reported in the literature (Menter, 1994; Pope, 2000) and therefore its details are not reported here.

\subsubsection{Computational Details}

The computed domain (Figure 3) is composed of two separated parts connected by the twin orifice plate that is $1.5 \mathrm{~mm}\left(0.15 D_{e}\right)$ thick. The upstream part of the domain had XYZ dimensions of $10 \times 20 \times 22 D_{e}$ and the downstream part $29.85 \times 20 \times 22 D_{e}$, respectively. Just one half of the domain in the $Y$ direction was modeled, due to the symmetry of the problem. Since the twin orifice plate had a finite thickness $(1.5 \mathrm{~mm})$, the inlet plane of the jet was set at $X=-0.15 D_{e}$ and the outlet plane of the jet at $X=0$ (see Figure 3a). The boundary condition for the inlet in the numerical simulation were given at the inlet plane of the upstream part of the domain, a uniform 
velocity of $0.0164 \mathrm{~m} / \mathrm{s}$ and a turbulence intensity of $2 \%$ were set on this plane. In the symmetry plane $(X Z)$ the symmetry boundary condition was used and for the orifice plate, the wall boundary condition was used. Atmospheric pressure boundary condition was used on the other boundaries of the domain which were far enough from the orifices and, were not affected by the flow.

Fluent 13.0 was used for the numerical analysis. For pressure-velocity coupling the SIMPLE algorithm was used. A second order upwind scheme was used to calculate the convective terms in the equations, integrated with the finite volume method. Regarding the accuracy of the results we have imposed a convergence criteria of $10^{-5}$ for all the variables residuals.

For the first case (Figure 1b), a grid dependency study was carried out on four different meshes: 0.7, 1.5, 4 and 9 million cells. The results of the 0.7 and 1.5 million cells grids shows large variations compared with 4 and 9 million cells grids, whose values are close. In this context we chose the grid of 4 million cells for numerical simulation. The same procedure was applied for the case 2 (Figure 1c) on three different meshes $(1.8,5$ and 8 million polyhedral cells). The 5 million cells mesh was chosen for the numerical simulation.

A mesh of 4 million Cartesian non-uniform cells was created for the case 1 and of 5 million polyhedral non-uniform cells for the case 2. The type of the mesh (Cartesian or polyhedral) was selected as a function of the lobe-shape. We believe that in the case of a rounded lobe (case 2), the polyhedral mesh follows better the curved line of the lobe than Cartesian grid.

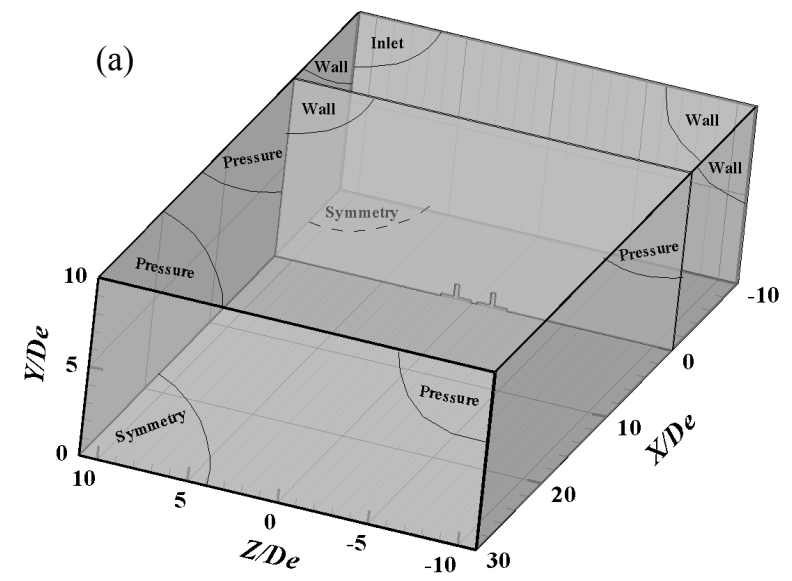

(b)

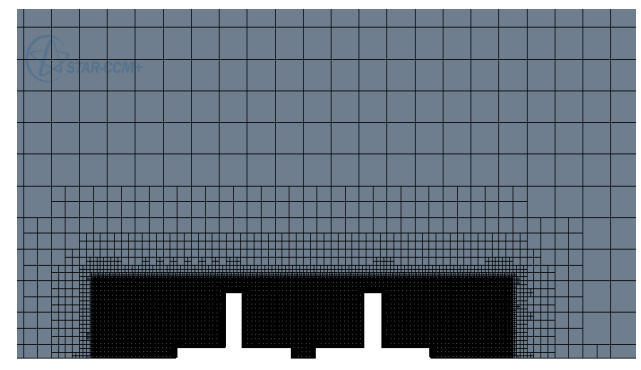

(c)

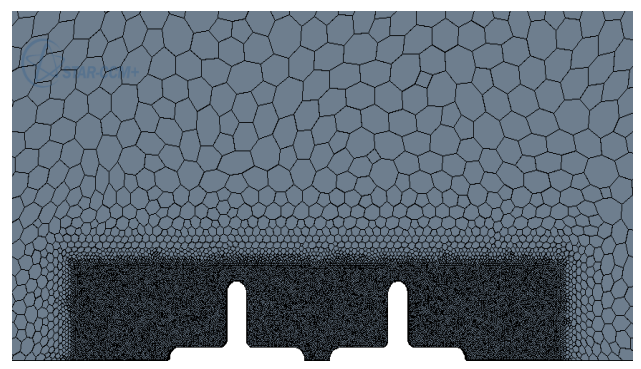

Figure 3. (a) The interest domain and the imposed boundary conditions; (b) A grid section on the orifice plate for Case 1 (LL O1); (c) A grid section on the orifice plate for Case 2 (LL O2)

The meshes were highly refined in the orifice thickness and on the outlet orifice area. The dimension of the smallest and largest cells in the orifice thickness was $0.02 \mathrm{~mm}$ and $0.12 \mathrm{~mm}$, respectively, being divided into 16 cells. On the outlet orifice plane, the smallest grid cell was $0.12 \mathrm{~mm}$ thick, and the largest one, which is far away from the orifice, was $2 \mathrm{~mm}$ thick. The values of $y+$ for the first gridline off the surfaces of the plate were less than 4 in the two cases, which is acceptable for the low Reynolds number formulation of the considered model.

Numerical simulations were performed on a system Intel Xeon, dual processor, six core, dual thread, $2.66 \mathrm{GHz}$ with 96GBRAM. For each case studied in this paper, 24 nodes were used. The time needed to reach convergence for each case was approximately eight days. 


\section{Results and Discussion}

The purpose of this study was on one hand to verify if we can achieve through numerical simulation a good reproduction of the phenomenon described in Figure 2 and described in the introductive part of this paper, and on the other hand, to evaluate the influence of this phenomenon on the dynamic parameters of the flow. We are comparing the dynamic quantities which are globally well predicted by the turbulence model SST $k-\omega$ (Meslem et al., 2011).

\subsection{Qualitative Analysis on the Influence of the Lobe Shape}

The velocity distributions on transverse planes at several axial distances $X$ between $0.5 D_{e}$ and $3 D_{e}$ were represented in Figure 4. In order to define the border of the jet we imposed a criterion of $U \geq 0.1 \mathrm{~m} / \mathrm{s}$.

Related to the orifice's geometry, locally we can observe different behaviors. Near the exit plane at $X=0.5 D_{e}$ and $X=1 D_{e}$, the same phenomenon, observed previously on the flow images in Figure 2, is found again. The right angled lobes (Figure 4a) of the jet are "pinched off, while the rounded lobes (Figure 4b) preserve their shapes.

At the axial distance $X=2 D_{e}$, the «pinch off» effect is still visible on the corresponding flow.

At $X=3 D_{e}$, the trace of the «pinch off» is only visible in the internal boundary layer (the region situated between the two jets). This first result reveals that the chosen grids allow a good representation of the local phenomena that we are willing to study. We have to elucidate now if these local phenomena have an influence on the dynamic quantities of the flow.
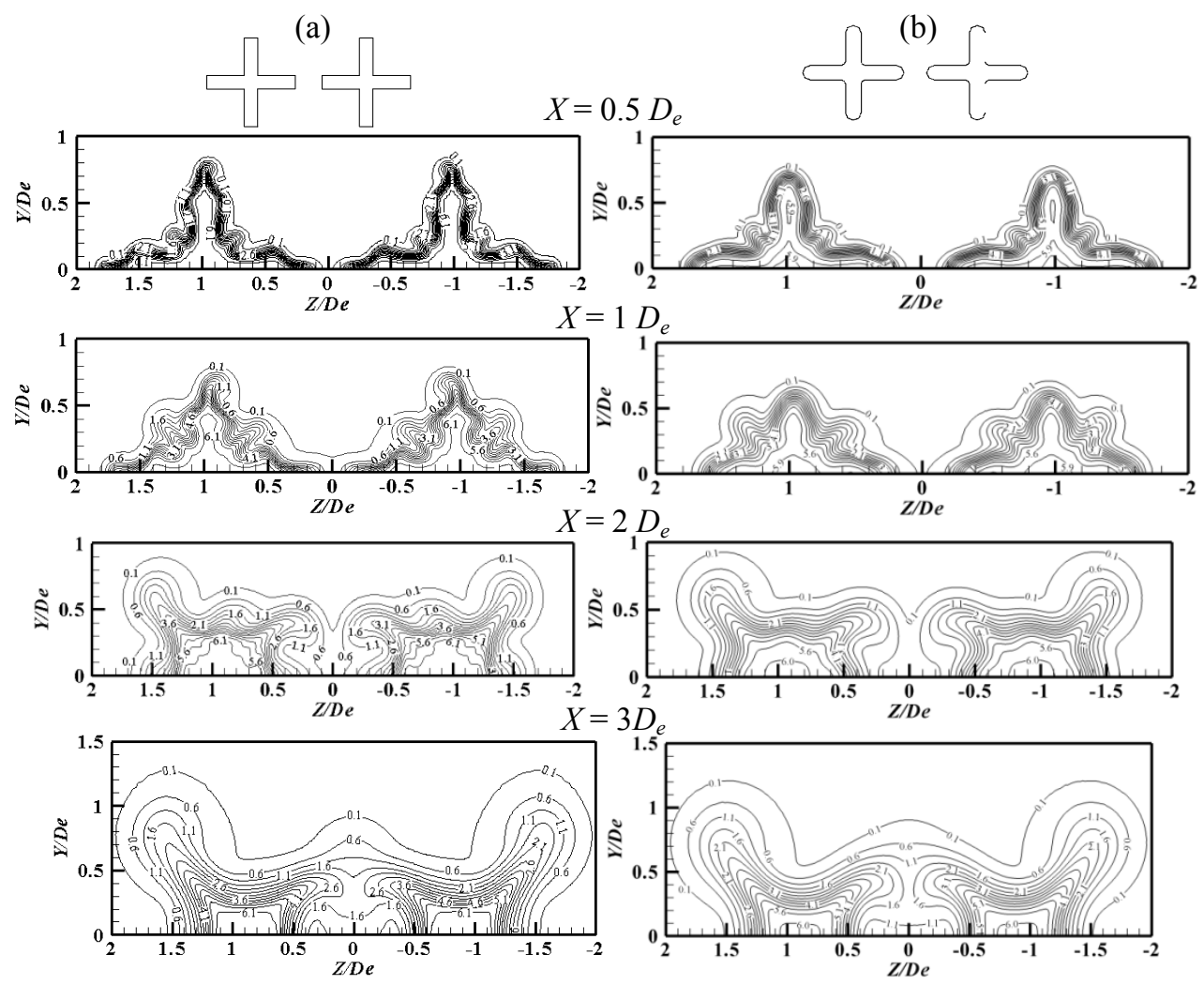

Figure 4. Streamwise velocity contours $U(\mathrm{~m} / \mathrm{s})$ in the transverse planes: (a) LL O1, (b) LL O2

The "pinch off" effect, at the lobe peaks, is also visible in Figure 5 giving the transverse velocity distributions at the axial distance $X=0.5 D_{e}$ and $X=1 D_{e}$. It can also be observed for the vertical right angled lobes, a stretching and an incurvation of the streamwise structures at the peaks of the lobes, which are governed by the depression created by the interaction of the two jets. 
(a)

(b)
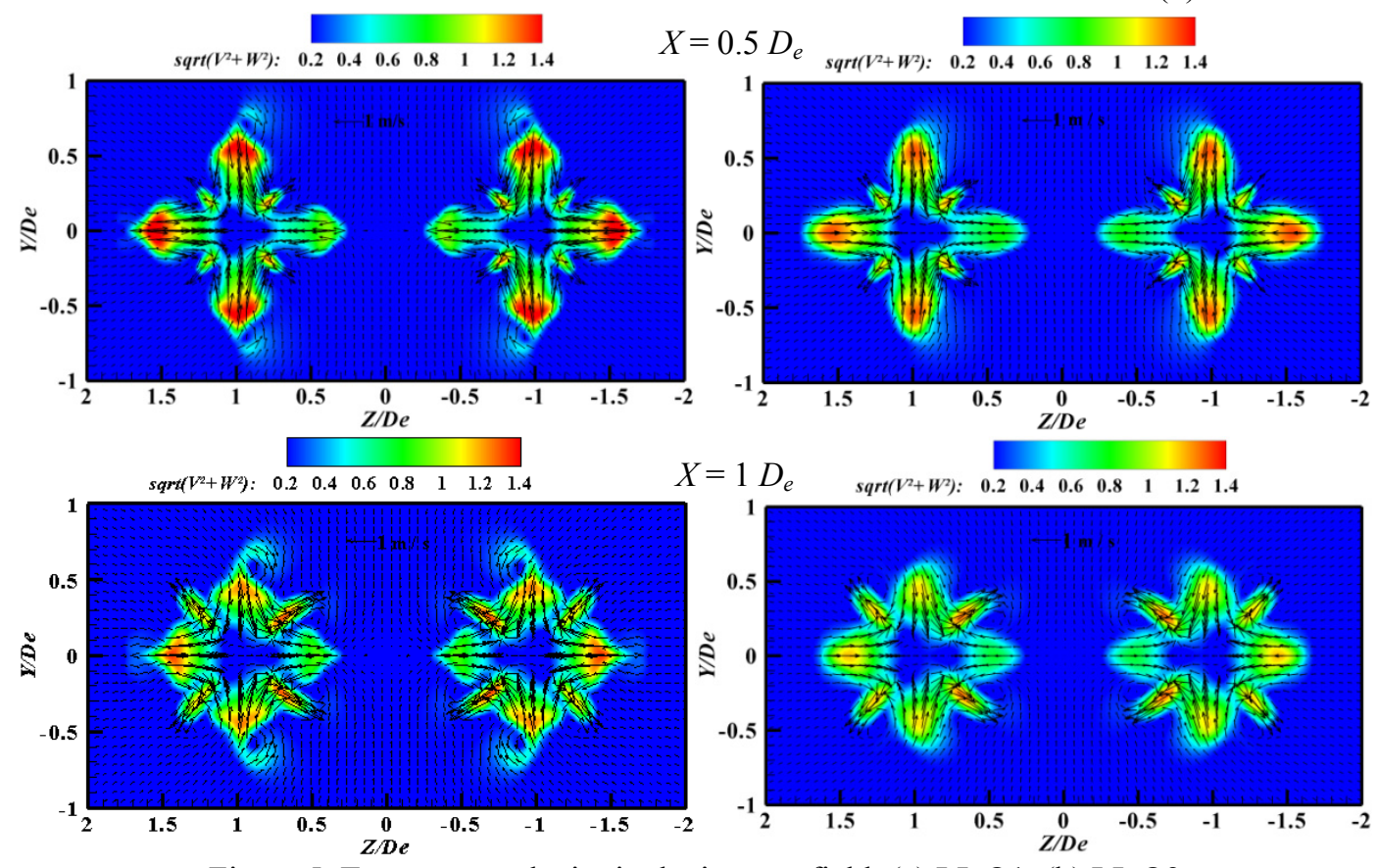

$X=1 D_{e} \quad \operatorname{sqrt}\left(V^{2}+W^{2}\right): \quad 0.2 \quad 0.4 \quad 0.6 \quad 0.8 \quad 1 \quad 1.21 .4$

Figure 5. Transverse velocity in the jet near field: (a) LL O1, (b) LL O2

(a)

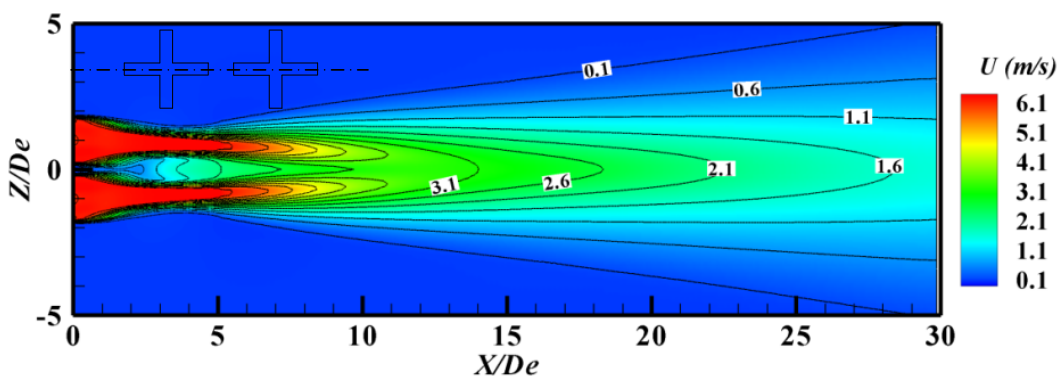

(b)
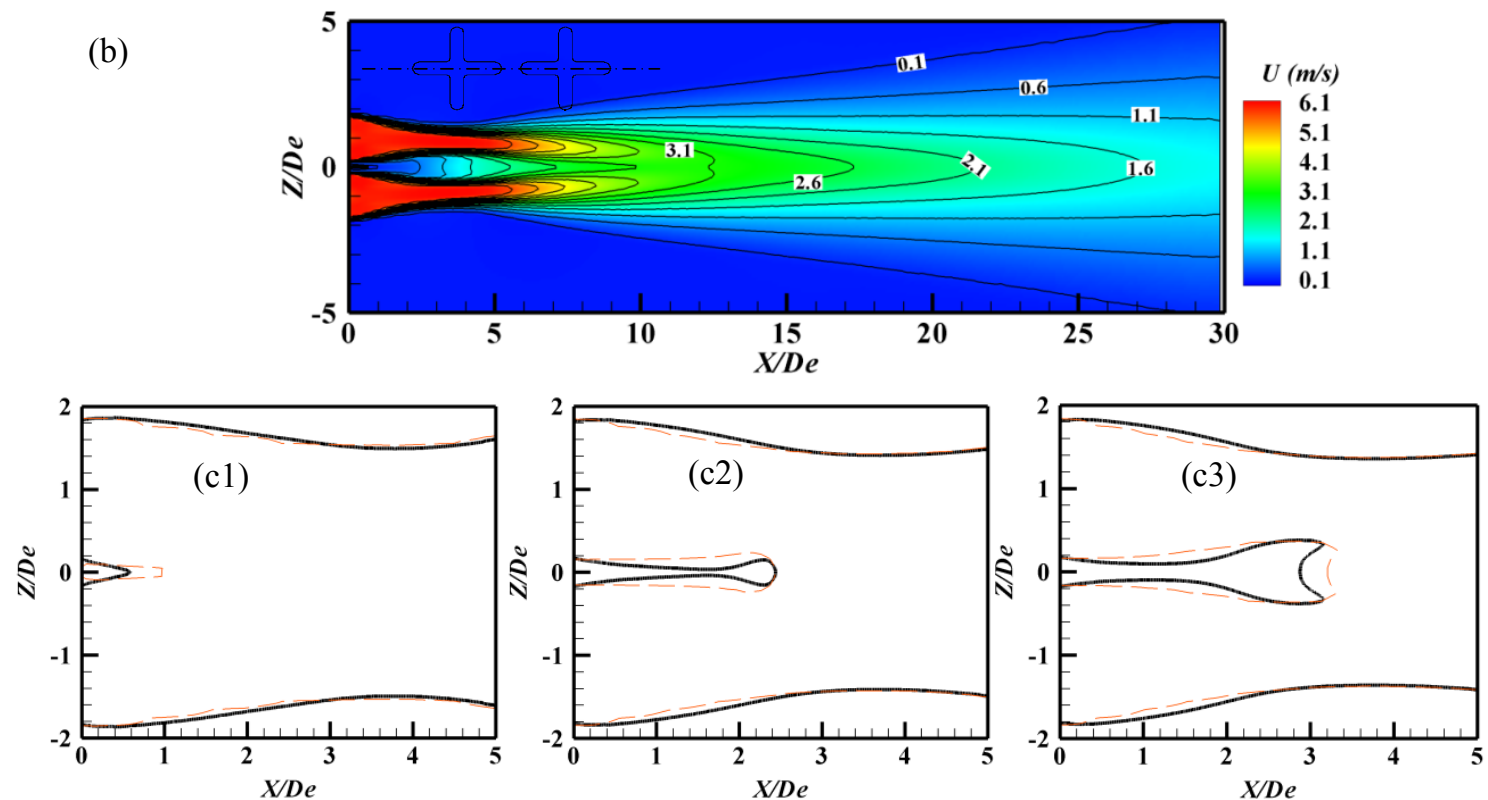

Figure 6. Isocontours of the streamwise velocity $U$ in the symmetry plane (XZ): (a) LL O1, (b) LL O2,(c) Comparison of $U$-isocontours in the first five equivalent diameters, Solid line for LL O1 and Dashed line for LL O2, (1) $U=0.1 \mathrm{~m} / \mathrm{s}$, (2) $U=0.6 \mathrm{~m} / \mathrm{s}$, (3) $U=1.1 \mathrm{~m} / \mathrm{s}$ 
Qualitatively, we can observe on the velocity fields in Figures 4 and 5, a larger dynamic expansion of the flow in the case where the lobes are rounded. The flow is more expanded and less pinched in the case LL O2. On the streamwise velocity fields from the symmetry plane $(X Z)$ from Figure 6, the "pinch off" effect is also visible, more particularly in the initial region $X \leq 5 D_{e}$. The superposition of iso-velocity contours for $U=0.1,0.6$ and $1.1 \mathrm{~m} / \mathrm{s}$ in this region, for the two configurations, allows highlightening this effect.

From the profiles given in Figure 7a, and from Table 1, it can be observed that for the same initial volumetric flow rate, the flow is more accelerated at the exit plane (i.e. initial jet centreline velocity $U_{0 J C}$ and initial maximum velocity $\mathrm{U}_{0 \mathrm{~m}}$ display larger values) for the LL O1 orifice compared to the LL O2 orifice. As is visible on the velocity fields in Figures 4 and 5, the right angles of the lobes lead to a compression of the flow at the jet exit plane.

On the transverse velocity $\mathrm{W}$ profiles given in Figure 7(b), the right angles of LL O1 orifice have an effect of local shear opposing to the entrainment at the lobe locations.
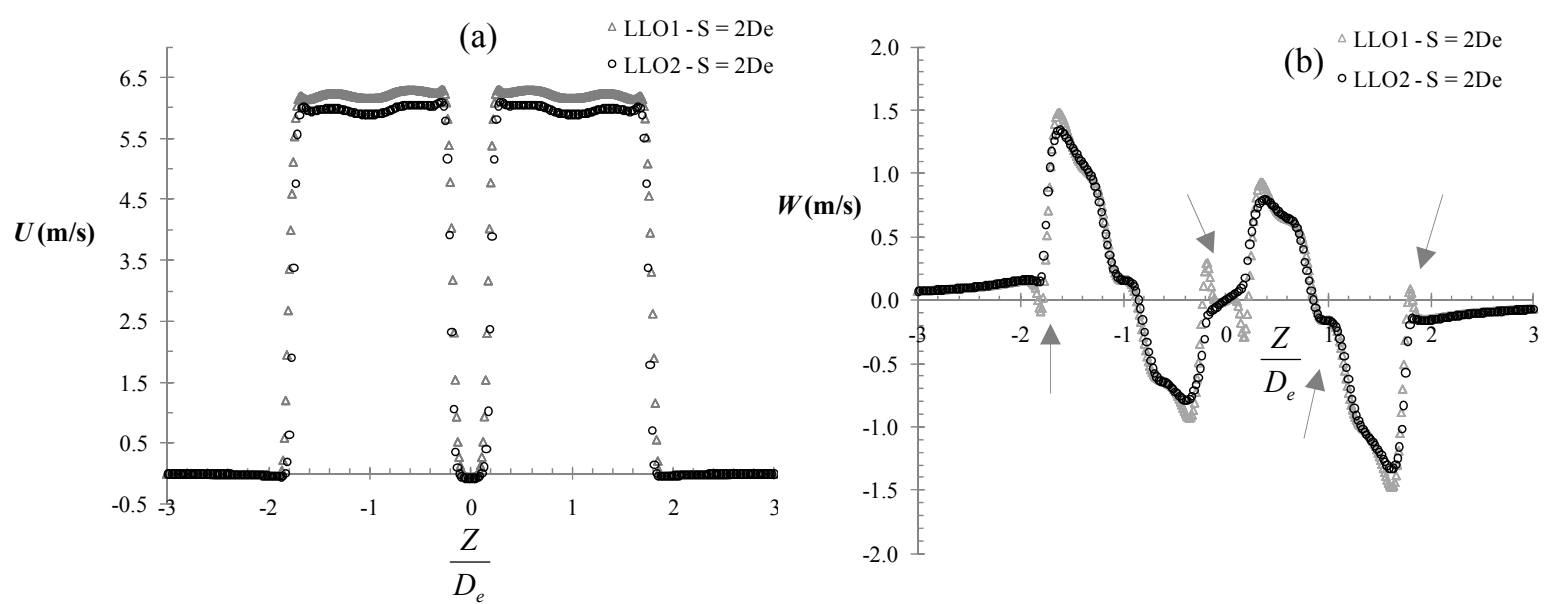

Figure 7. Streamwise velocity profiles (a) and transverse velocity profiles (b) in the symmetry plane $(X Z)$ at $X=$ $0.25 D_{e}$. Arrows are for designing local shear due to lobes corners in the case of LL O1

Prior to a quantification of the consequences of the shape of the lobes on the dynamic quantities, we wanted to observe the fields of the streamwise vorticity in the transverse planes in the near exit region. The objective was to identify the physical link between the observed "pinch off" effect of the lobes observed and the subsequent organization of the streamwise vorticity.

We represented in Figure 8 the comparison of the streamwise component of the rotational field (Eq. 3) in the transverse plane at the streamwise distances $0.5 D_{e}$ and $1 D_{e}$.

$$
\omega_{X}=\left(\frac{\partial W}{\partial Y}-\frac{\partial V}{\partial Z}\right) \frac{D_{e}}{U_{0 J C}}
$$


(a)
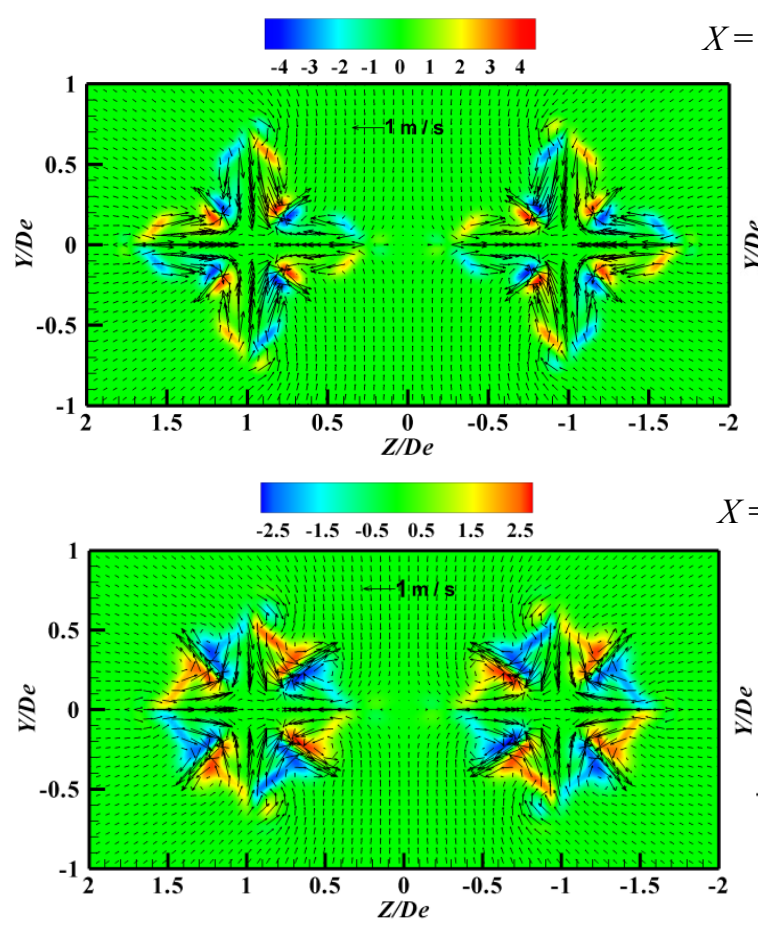

(b)

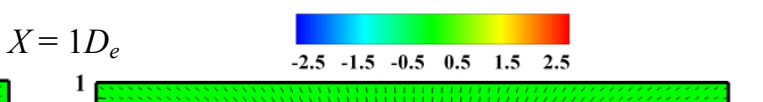

Figure 8.Streamwise axial vorticity $\omega_{X}$ contours in the jet near field: (a) LL O1, (b) LL O2

At the axial distance $X=0.5 D_{e}$, for the right angled lobes (Figure 8a), besides the more pronounced inclination of the vortical pairs at the peak of each lobe (corresponding to the previously observed "pinch off" effect), we are observing additional traces of vorticity, hardly observable with the chosen scale for $\omega_{x}$. In order to put in evidence this phenomenon, we represented in Figure 9 the same fields of vorticity (at $X=0.5 D_{e}$ ) with a reduced scale for $\omega_{x}$. This way, supplementary counter rotating vortex pairs are observable. Their intensity is weaker and their rotation sign is opposed to the pair of vortices corresponding to the lateral sides of the lobes. As it can be seen in Figure 9, additional "real" pairs of vorticity, are formed only for the horizontal lobes. At the vertical lobes, additional vorticity is present in the form of only one vortex, due to the depression in the region between the two jets which is reducing the counter rotating vortical pair in a unique structure inclined towards the inter-orifice region. This observation agrees with the previous remarks related to the transverse velocity fields (Figure 5). These additional structures are not advantageous for the entrainment process as their rotation is locally opposing the main induction phenomenon at the peak of the lobe. We have to recall here the fact that in the lobed orifice jet the entrainment is produced at the lobe peaks and fluid ejections take place in the lobe troughs (Nastase, et al., 2008; Meslem, et al., 2011).

(a)

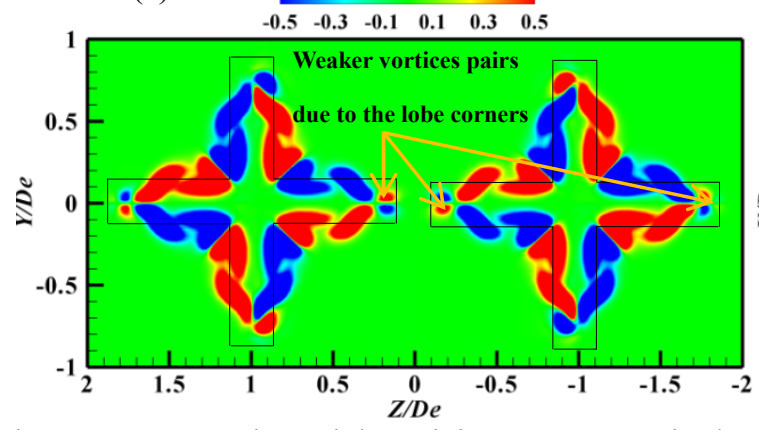

(b)

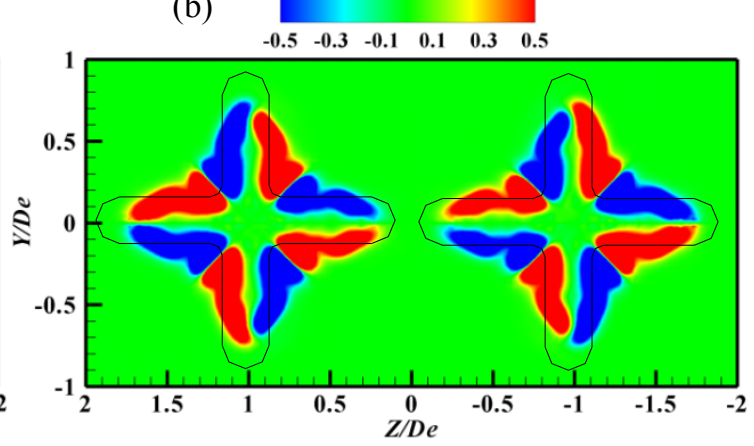

Figure 9. Streamwise axial vorticity $\omega_{\mathrm{x}}$ contours in the transverse plane $X=0.5 D_{e}$ with a reduced isocontours range for a visualization of the weaker vortices pairs: (a) LL O1, (b) LL O2 
At the following streamwise positions, which are not presented here, the additional vorticities are disappearing progressively, their traces being identifiable through the stretching of the remaining structures and their slight intensification. This effect of intensification of the local vorticity is visible in Figure 10, where we are presenting the axial evolution of the maximum value of the streamwise vorticity (the maximum difference is $14 \%$ at $X=1.5$ $D_{e}$ ). We have to point here an important observation: the increase of the streamwise vorticity level is not necessary corresponding to an intensification of the induction. The increase of the streamwise vorticity in the case LL O1 is resulting from the "pinch off" effect due to the additional vorticity generated by the lobe corners (see Figure 9).

As for the «pinch-off» effect, created by the right angles of the orifice, we searched in the literature for similar phenomena. Quinn (1992), Grinstein and DeVore (1996), Chua et al. (2001) and Wang et al. (2003) confirmed the existence of a switching-over phenomenon in certain square jets. Quinn (1992) showed that the flow field of the square jet was characterized by eight streamwise vortices. Theses streamwise vortices are organized as sketched in Figure 11(a), in four pairs of vortices (each located at one corner) occurring close to the nozzle at $X=$ $0.3 D_{e}$. The fluid between the two vortices of a pair is induced inward. For ease of identification, such a vortex pair is referred to as the "inflow pair" and conversely, an "outflow pair" will be in the opposite sense. These inflow vortices would result in a contraction of the jet cross-section in the diagonal-directions.

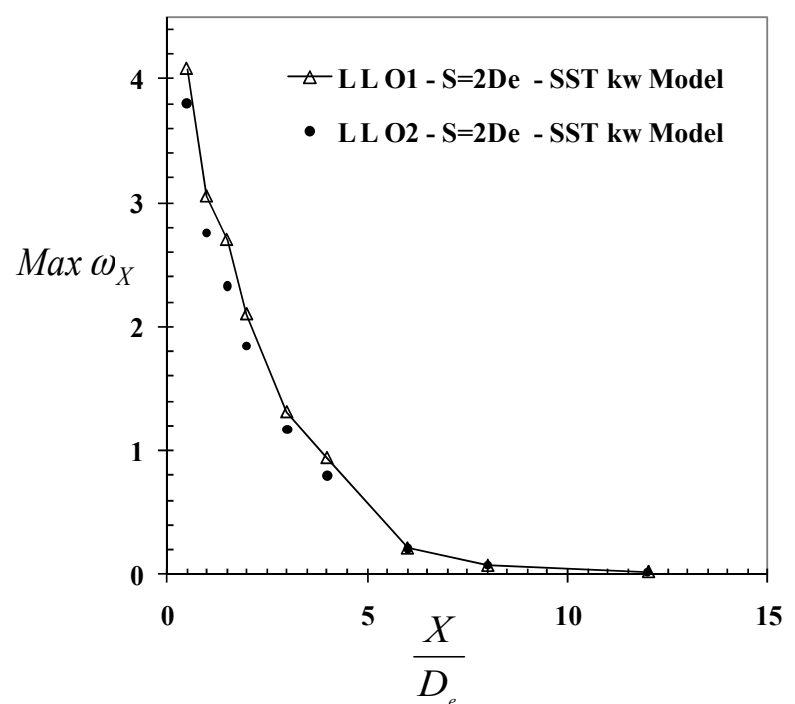

Figure 10. Maximum streamwise vorticity $\omega_{X}$ comparison

In a jet flow generated by a cross-shaped orifice, having right angled lobes, we were expecting to obtain at the peaks of the lobes the same phenomenon as the one observed by (Quinn, 1992) in the square jet. As illustrated in Figure 11(b), on each peak of the lobe, instead of two pairs of vortical structures only one is formed. We are making a hypothesis, which is not yet demonstrated, that the width "a" of the lobe (or of a rectangle) is conditioning the organization of the vorticity around the right angles. It must exist a critical width "ac" for which the distance between the two angles is too small for the development of all four structures. In this case, the two external structures are disappearing and the internal structures are forming a pair of vortices on the type of "outflow pair" which is the origin of the streamwise structure observable at the peak of each lobe with right angles of the orifice $\mathrm{O} 1$ (Figure 2b). This remark calls the perspective of the analysis of the mixing performance for the lobed jet generated by the cross $\mathrm{O} 1$ as a function of the lobe width.

As for the organization of the streamwise structures in the twin jet of the LL O1 configuration (Figure 11c), a complementary phenomenon occurs and modifies the previously observed organization of the free jet generated by $\mathrm{O} 1$. It is the depression created between the two jets, acting on the vorticity at the peaks of the vertical lobes. The pairs of vortices at the lobe peaks are reducing in one structure oriented towards the inner part of the convergence region (Figure 9a) given the effect of depression.

\subsection{Quantitative Analysis on the Influence on the Shape of the Lobe}

We are comparing in Figure 12a, the streamwise evolution of the transverse surface of the flow defined at each streamwise distance $X$ by: 


$$
A_{\text {Flow }}=\left.\int d A\right|_{U>0.15 \mathrm{~m} / \mathrm{s}}
$$

In this figure we are observing a difference (with a maximum of 7\%) which might be considered as being non important from the point of view of the scale of two coalescent jets, but for the scale of a perforated panel (Figure 1a) used for air diffusion in mixing ventilation systems (Meslem et al., 2010), the gain in expansion of the multi-jet configuration might be non negligible. In the same way, on the streamwise evolution of the volumetric flow rate (Figure 12b), defined at each streamwise distance $\mathrm{X}$ by:

$$
Q=\left.\int U d A\right|_{U>0.15 m / s}
$$

We are observing a slight increase of the values corresponding to the case with rounded lobes (with a maximum difference of $3.5 \%$ ).

The streamwise evolution of the mean radius of the flow (Figure 12c) defined by:

$$
r_{0.1 U_{m}}=\sqrt{\frac{\left.\int d A\right|_{U>0.1 U_{m}}}{\pi}}
$$

shows, as in the case of $A_{\text {Flow }}$, a slight advantage for the case with rounded lobes, on the order of $5 \%$, in the far region. As for the mean velocity decay (Figure 12d), given by:

$$
U_{\text {mean }}(X)=Q(X) / A_{\text {Flow }}(X)
$$

the gap between the two curves is not sensible, leading to similar jet throws for both configurations.

(a)

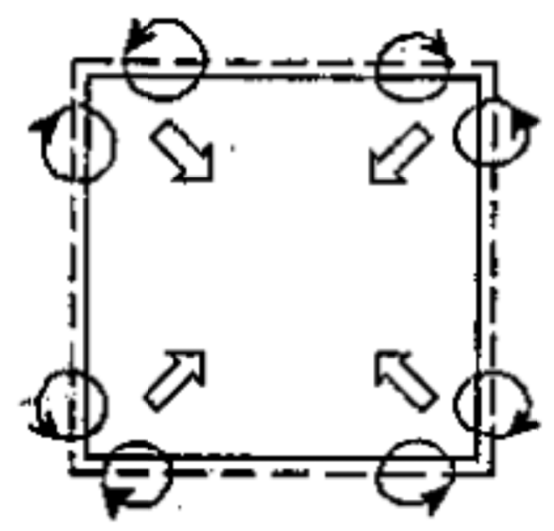

(b)

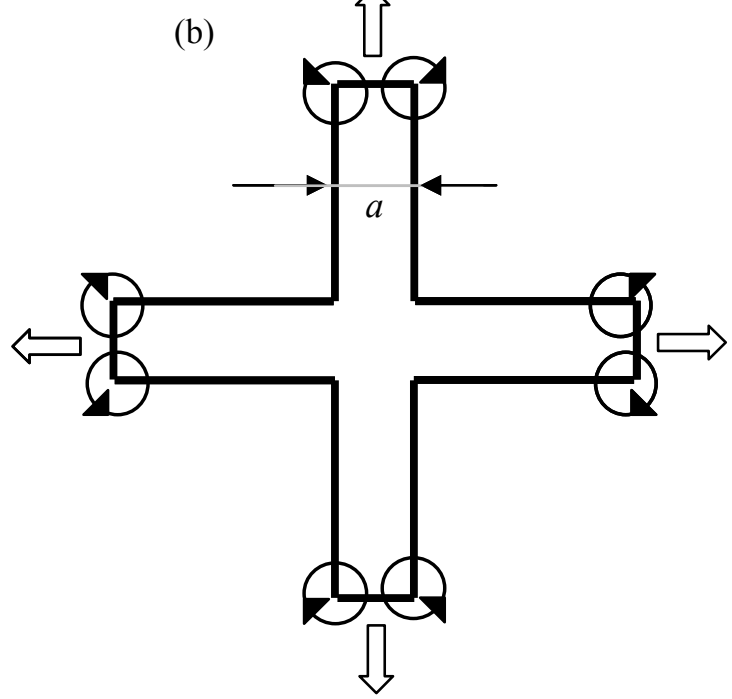

(c)

Subatmospheric region $(U<0)$

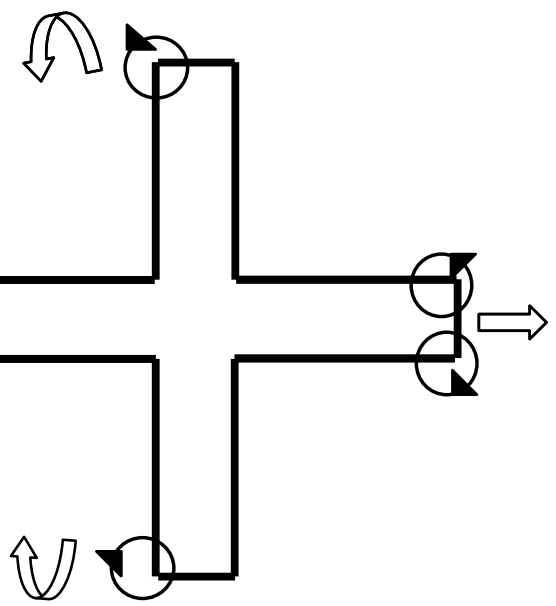

Figure 11. Schematic of the mean streamwise vortex distributions and the corresponding effects in the square jet (Quinn, 1992; Wang et al., 2003) (a), in the cross-shaped jet (b) and in the twin cross-shaped jet (c) 

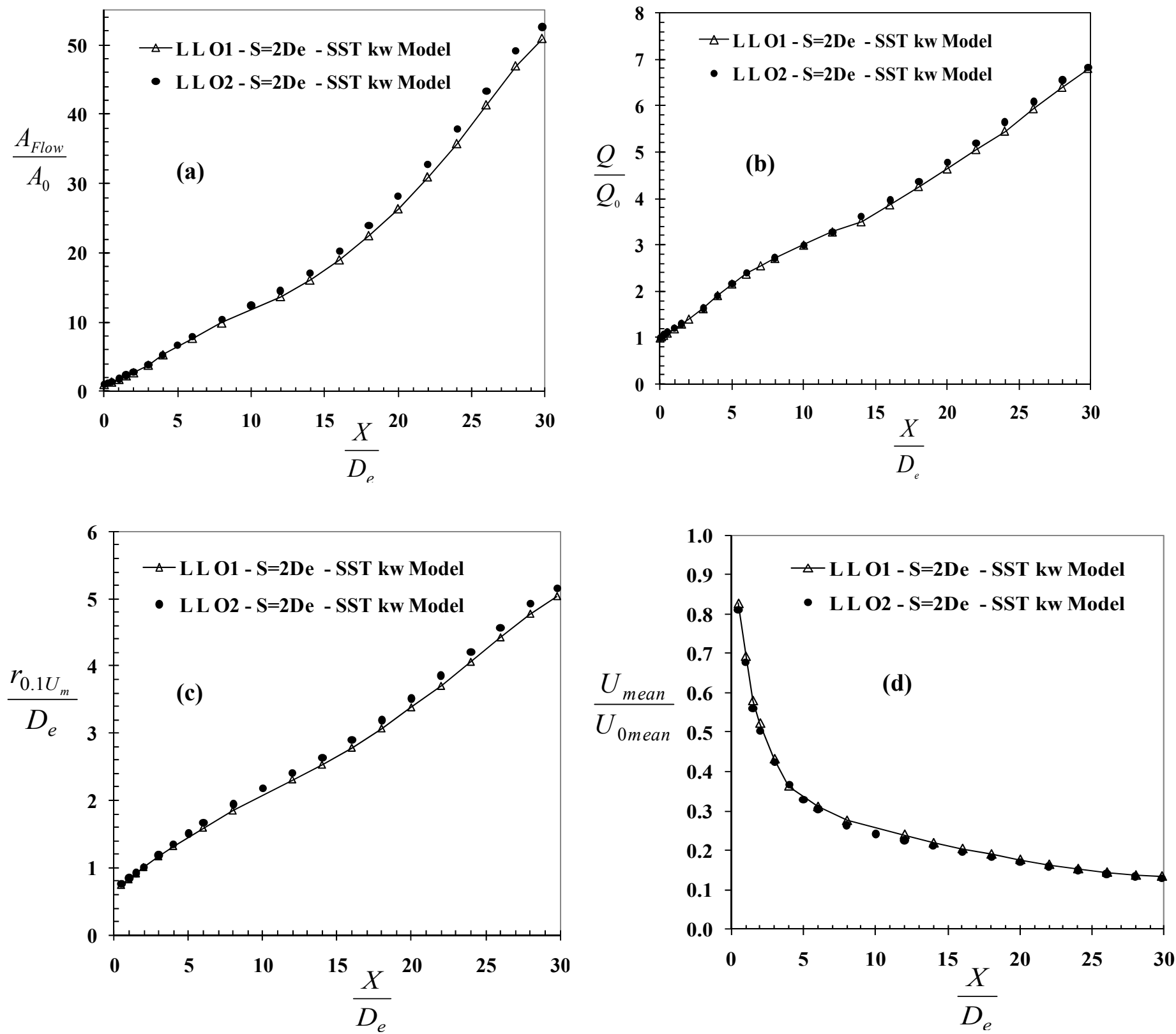

Figure 12. Streamwise evolution of flow area (a), twin jet volumetric flow rate (b), Flow radii (c) and Flow mean velocity (d)

Let us observe now the consequences of the previous differences on the turbulent fields. Figure 13 is presenting the Turbulent Kinetic Energy (TKE) fields, superposed on the transverse velocity fields, at the axial distances $X=$ $0.5 D_{e}$ and $X=1 D_{e}$. 
(a)

(b)

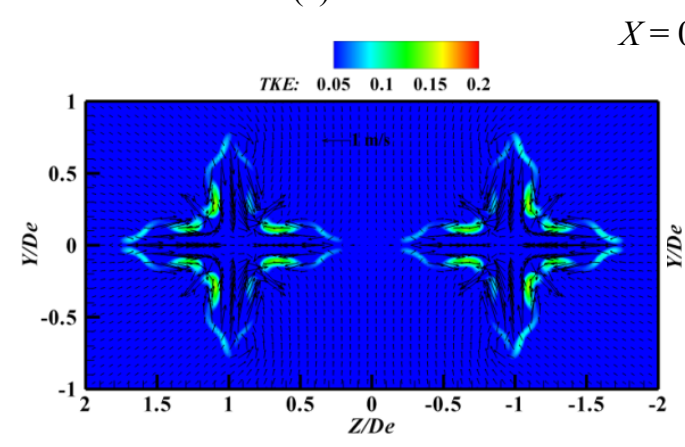

$X=0.5 D_{e}$
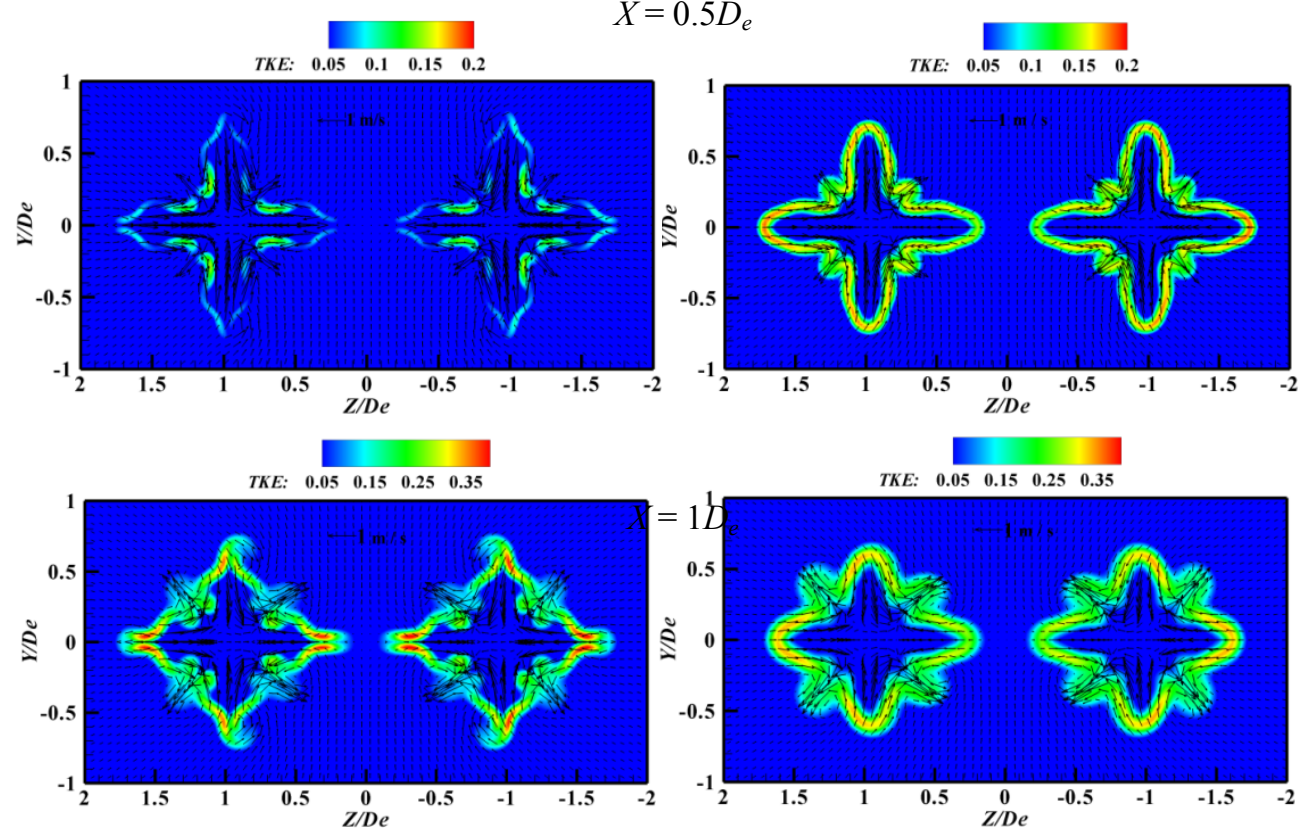

Figure 13. Isocontours of the TKE in the jet near field: (a) LL O1, (b) LL O2

For the configuration LL O2 (with rounded lobes) the turbulence is less concentrated than for LL O1 and occupies more extended surface. The characteristic "pinch off" effect is found once again for the right angled lobes in the LL O1 configuration. For this configuration the higher levels of TKE are corresponding to the additional vortices due to the lobe corners (Figure 9). The transverse velocity field, superposed to the $T K E$ field is clearly highlighting the rotation of the flow towards the inter-jet region at the peaks of the vertical right angled lobes. This movement is generated by the vortex that was put in evidence previously, in Figure 9a, which is generated through the coalescence of one vortex pair in a unique vortex due to the influence of the depression created on its turn by the merging of the two jets.

On the Average Turbulent Kinetic Energy ATKE (Figure 14), defined by Eq. (8), we are observing some differences related to the previous observations. For $X \leq 1 D_{e}$, this quantity is slightly superior when the lobes are rounded (LL O2), as for the larger development of the streamwise structures (which are not blocked or stretched by any additional vortices, as in the case of LL O1). After this distance, the turbulence is weaker in the case LL $\mathrm{O} 2$, given the increase in entrainment (greater dilution of the flow).

$$
\operatorname{ATKE}(X)=\frac{\left.\int U(Y, Z) \cdot \operatorname{TKE}(Y, Y) d Y d Z\right|_{U>0.15 \mathrm{~m} / \mathrm{s}}}{\left.\int U(Y, Z) d Y d Z\right|_{U>0.15 \mathrm{~m} / \mathrm{s}}}
$$




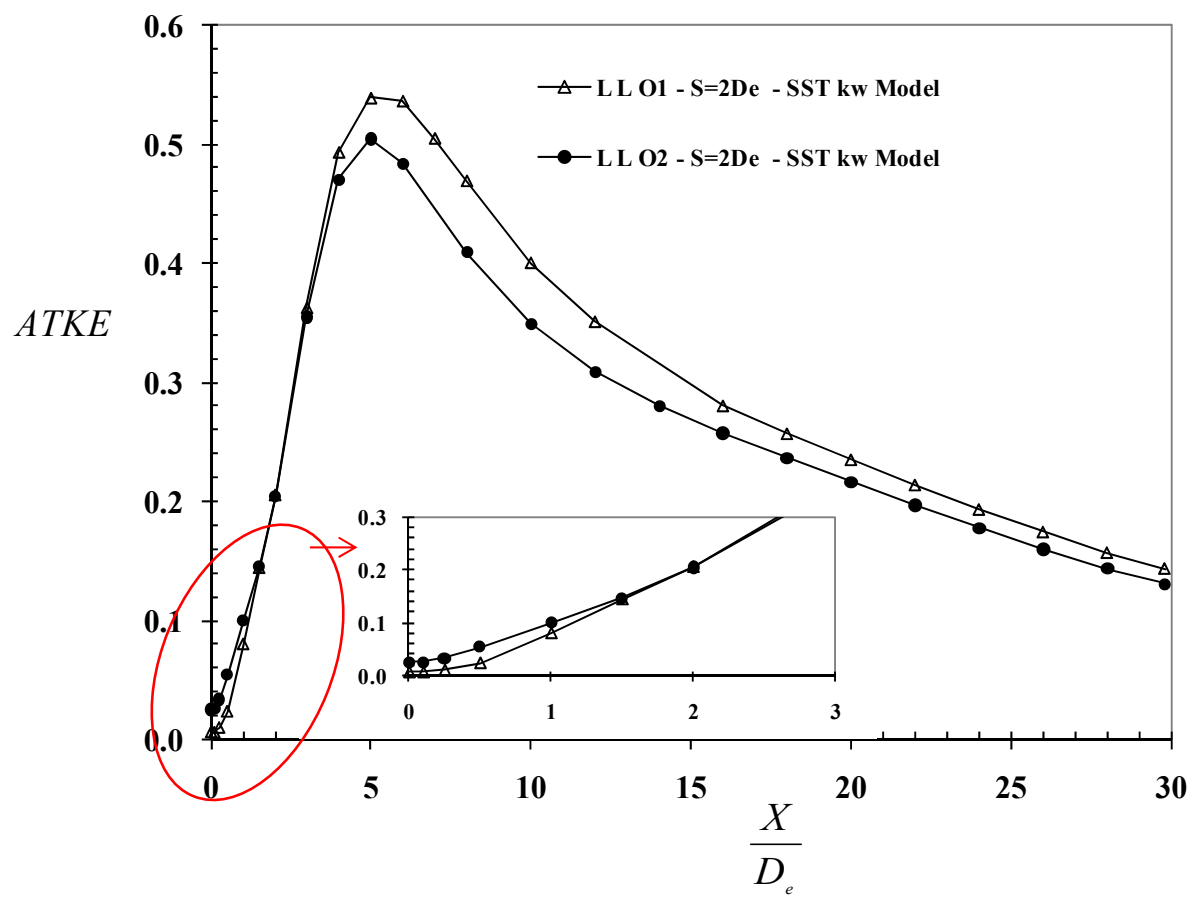

Figure 14. Averaged Turbulent Kinetic Energy comparison

\section{Conclusion}

In this paper we studied by numerical means the influence of the lobe shape for cross-shaped orifices, on the dynamic quantities of the flow composed by two coalescent jets. It was shown that the lobes with rounded peaks are more advantageous than the right angled lobes. The recorded gain in terms of global quantities is of $7 \%$ for the global expansion of the flow and of $3.5 \%$ for the volumetric flow rate. If these differences are rather small, it is not advisable to ignore them, due to the fact that is possible that the real gains be more important as the predicted ones. Indeed, the confrontation between the SST k- $\omega$ model and experimental data, proposed in our previous works, brought us to revise our expectations related to the numerical prediction using RANS models. The "best" turbulence model is able, in a parametric optimization approach, to give an indication of the way of variation for the dynamic quantities of the flow but does not provide accurate results for these variations. In addition, local slight values of gain in induction can lead to a significant improvement of the air diffusion and thermal comfort of the users when a lobed perforated panel is used. The exploration of these aspects of coupling between jet flow and environment can now be experimentally studied in the full-scale model room with controlled thermal environment recently constructed at LaSIE Laboratory.

On the local phenomena which have been explored numerically, a comparison of the streamwise vorticity and of the transverse velocity of the two flows (from right angled lobed orifices and rounded lobed orifices) allows an understanding of the phenomena responsible for the recorded differences between the two flows. The obvious correlation between the calculated fields and the experimental flow visualizations reinforces our opinion related to the capability of RANS turbulence models to meet the needs of a qualitative exploration of turbulent flows.

Finally, the slight increase in induction which has been observed is accompanied by an increase of the average turbulent kinetic energy, in the near exit region, followed by a reduction of it beyond two equivalent diameters, due to a higher dilution of the flow.

\section{Acknowledgement}

This study was carried out as part of the VaicteurAir2 project, funded by OSEO.

This work was partially supported by the grants of the Romanian National Authority for Scientific Research, CNCS - UEFISCDI, project numbers: PN-II-PD-PCE-2011-3-0099 and PN-II-ID-PCE-2011-3-0835.

\section{References}

Chua, L. P., Li, Y. F., Yu, C. M. S., \& Zhou, T. (2001). Axis-switching of a square jet. 14th Australian Fluid Mechanics, Australia., Adelaide University. 
Croitoru, C. (2011). Theoretical and experimental studies regarding the influence of air turbulence intensity inside buildings on thermal comfort, $\mathrm{Ph}$. D. Thesis, Technical University of civil Engineering, Bucharest.

Croitoru, C., Nastase, I., Iatan, A., Iordache, V., Meslem, A., \& Colda, I. (2011). Numerical and experimental modeling of airflow and heat transfer of a human body. Roomvent 2011, Trondheim, Norway.

Grinstein, F. F., \& DeVore, C. R. (1996). Dynamics of coherent structures and transition to turbulence in free square jets. Phys. Fluids, 8, 1237-1251. http://dx.doi.org/10.1063/1.868895

Menter, F. R. (1994). Two-equation eddy-viscosity turbulence models for engineering applications. AIAA Journal, 32(8), 1598-1605. http://dx.doi.org/10.2514/3.12149

Meslem, A., Diaa, A., Begheina, C., El-Hassana, M., Nastaseb, I., \& Vialle, P. J. (2011). A comparison of three turbulence models for the prediction of parallel lobed jets in perforated panel optimization. Building and Environment, 46, 2203-2219. http://dx.doi.org/10.1016/j.buildenv.2011.04.037

Meslem, A., El-Hassan, M., \& Nastase, I. (2011). Analysis of jet entrainment mechanism in the transitional regime by time-resolved PIV. Journal of Visualization, 14(1), 41-52. http://dx.doi.org/10.1007/s12650-010-0057-7

Meslem, A., Nastase, I., \& Allard, F. (2010). Passive mixing control for innovative air diffusion terminal devices for buildings. Building and Environment, 45, 2679-2688. http://dx.doi.org/10.1016/j.buildenv.2010.05.028

Nastase, I., Meslem, A., \& Gervais, P. (2008). Primary and secondary vortical structures contribution in the entrainement of low Reynolds number jet flows. Experiments in Fluids, 44(6), 1027-1033. http://dx.doi.org/10.1007/s00348-008-0488-2

Nastase, I., Meslem, A., Vlada, I., \& Colda, I. (2011). Lobed grilles for high mixing ventilation - An experimental analysis in a full scale model room. Building and Environment, 46(3), 547-555. http://dx.doi.org/10.1016/j.buildenv.2010.08.008

Pope, S. B. (2000). Turbulent flows, ed. C.U.P. Cambridge. http://dx.doi.org/10.1017/CBO9780511840531

Quinn, W. R. (1992). Streamwise evolution of a square jet cross-section. AIAA Journal, 30, 2853-2857. http://dx.doi.org/10.2514/3.48973

Wang, X. K., Chua, L. P., \& Yu, S. C. M. (2003). On the near-field of a square jet with vortex-generating tabs. Fluid Dynamics Research, 32, 99-117. http://dx.doi.org/10.1016/S0169-5983(03)00015-7 